\title{
VOLUMES OF IMAGES OF VARIETIES IN PROJECTIVE SPACE AND IN GRASSMANNIANS
}

BY

\author{
H. ALEXANDER
}

\begin{abstract}
If $V$ is a complex analytic subvariety of pure dimension $k$ in the unit ball in $\mathbf{C}^{n}$ which does not contain the origin, then the $2 k$-volume of $V$ equals the measure computed with multiplicity of the set of $(n-k)$-complex subspaces through the origin which meet $V$. The measure of this set computed without multiplicity is a smaller quantity which is nevertheless bounded below by a number depending only on the distance from $V$ to the origin. As an application we characterize normal families in the unit ball as those families of analytic functions whose restrictions to each complex line through the origin are normal. The complex analysis which we shall need will be developed in the context of uniform algebras.
\end{abstract}

1. Introduction. Let $V$ be a pure $n-1$ dimensional analytic subvariety of the unit ball $B_{n}$ in $\mathbf{C}^{n}$ which does not contain the origin. By a result of Kneser [9] and Lelong [10], the $2(n-1)$-volume of $V$ in $C^{n}$ is equal to the measure, computed with multiplicity, of the image $V$ in complex projective space $\mathrm{P}^{n-1}$ (under the restriction to $V$ of the natural projection $\rho: \mathbf{C}^{n} \backslash\{0\} \rightarrow \mathrm{P}^{n-1}$ ) where the measure in $\mathbf{P}^{n-1}$ is the natural invariant measure appropriately normalized. In other words: the volume of $V$ is equal to the measure, counted with multiplicity, of the set of complex lines ( $=$ one dimensional complex linear subspaces of $\mathbf{C}^{n}$ ) in $\mathbf{C}^{n}$ which meet $V$. Now if $V$ stays near the origin, say $V$ meets the ball of a fixed radius $r$, then the $2(n-1)$-volume of $V$ is bounded below (by $(1-r)^{2(n-1)} \cdot \alpha(n-1)$ where $\alpha(k)$ is the volume of the unit ball in $C^{k}$, see [14]); consequently, the measure, with multiplicity, of the set of complex lines which meet $V$ is bounded below. If we consider the measure of the family of complex lines which meet $V$, not with multiplicity, but simply as a subset of $\mathbf{P}^{n-1}$, we get a smaller quantity which, as we shall prove, is still bounded below. The precise result is this: There exists a strictly positive, decreasing, continuous function $q(r)$, defined for $0<r<1$, such that if $V$ is a pure $n-1$ dimensional subvariety of $B_{n}$ and $r$ is the distance from $V$ to the origin, then the measure of the set (without reference to multiplicity) of complex lines in $\mathrm{P}^{n-1}$ which meet $V$ is bounded below by $q(r)$.

More generally, we shall consider subvarieties of $B_{n}$ of arbitrary pure dimension. The Grassmann manifold $G(n, k)$ of all $\mathbf{C}$-linear subspaces of $\mathbf{C}^{n}$ of complex dimension $k$ carries a natural measure which is invariant under unitary transfor-

Received by the editors September 19, 1972.

AMS (MOS) subject classifications (1970). Primary 32C30, 32E20, 46J10; Secondary 32A17, $53 \mathrm{C65}$.

Key words and phrases. Complex analytic variety, volume of variety, Grassmann manifold, normal family, uniform algebra. 
mations. We shall see that if $V$ is a pure $k$-dimensional subvariety of $B_{n}$ which does not contain the origin, then the $2 k$-volume of $V$ equals the measure, counted with multiplicity, of the set of elements of $G(n, n-k)$ which meet $V$ (when the measure in $G(n, n-k)$ has been suitably normalized). This result bears a resemblance to Crofton's formula in integral geometry, but differs in that only $n-k$ planes through the origin are involved. The proof, however, does invoke Crofton's formula which we shall state below. In analogy to the case of codimensional 1 , we study the measure, without multiplicity, of the set of elements of $G(n, n-k)$ which meet $V$ and show that it is bounded below by a number depending only upon the distance from $V$ to the origin.

As an application we derive a characterization of normal families of analytic functions in $B_{n}$ : A family $\Im$ of analytic functions on $B_{n}$ is normal if and only if the restriction of $\exists$ to every complex line through the origin is normal. This result is a "radial" analogue to a theorem of Nishino [11] who proved that a family $\rightarrow$ of analytic functions on a domain $\Omega$ in $\mathbf{C}^{2}$ is normal if the restriction to each coordinate line (of the form $z_{1}=\lambda$ or $z_{2}=\lambda$ ) is normal. A local version at the origin of the radial theorem also holds. A direct consequence is a classical theorem of Hartogs [7] to the effect that a formal power series at 0 in $\mathbf{C}^{n}$ is convergent if its restriction to each complex line through the origin is convergent. An elementary proof, valid for fields more general than the complex numbers, has recently been given by Stoll [13].

The complex analysis which we shall need will be carried out in the context of uniform algebras; here we shall follow the standard terminology of the books of Gamelin [5] and Stout [15]. One of our results is the following: Let $A$ be a uniform algebra with maximal ideal space $\mathcal{c} M$. Let $x_{0} \in \mathcal{O}$ and let $\sigma$ be a positive measure on $\mathcal{M}$ representing $x_{0}$. Let $f$ and $g$ belong to $A$ and have no common zeros on $\mathcal{C} M$ and suppose $f\left(x_{0}\right)=0$. Let $x \mapsto[f(x), g(x)]$ define a mapping of $\mathcal{M}$ into $\mathbf{P}^{1}$ (where $\left[z_{1}, z_{2}\right]$ are homogeneous coordinates). Then the image of $M$ under this mapping has measure in $\mathbf{P}^{\mathbf{l}}$ which is greater than or equal to $\int|f|^{2} /\left(|f|^{2}+|g|^{2}\right) d \sigma$. The uniform algebra results will be applied to certain algebras $P(K)$, where $K$ is compact in $\mathbf{C}^{n}$ and $P(K)$ denotes the uniform closure of the polynomials in the space $C(K)$ of all continuous functions on $K$. The sets $K$ will include one-dimensional varieties as a special case. The results on higher dimensional varieties will then be obtained by integral geometric arguments.

2. In this section we prove the following

Theorem 2.1. Let $V$ be a pure $k$ dimensional analytic subvariety of $B_{n}$, the open unit ball in $\mathbf{C}^{n}$, which does not contain the origin. Then the $2 k$-volume of $V$ is equal to the measure, counted with multiplicity, of the set of $n-k$ dimensional complex linear subspaces of $\mathbf{C}^{n}$ which meet $V$. More precisely: Define $n_{V}$ on the Grassmannian $G(n, n-k)$ of $n-k$ dimensional $\mathbf{C}$-linear subspaces of $\mathbf{C}^{n}$ by $n_{V}(J)=$ the number of points in $V \cap J$. Then

$$
2 k \text {-vol } V=\frac{\pi^{k}}{k !} \int n_{V}(J) d J
$$


where " $d J$ " is the invariant volume element on $G(n, n-k)$ normalized to have total mass one.

We shall identify $G(n, j)$ with $\tilde{G}(n-1, j-1)$, the set of $j-1$ dimensional complex planes in $\mathbf{P}^{n-1}$ in the usual way; namely, if $\rho: \mathbf{C}^{n} \backslash\{0\} \rightarrow \mathbf{P}^{n-1}$ is the natural projection, $J \in G(n, j)$ corresponds to $\rho(J) \in \tilde{G}(n-1, j-1)$. By a local variety in $\mathbf{P}^{s}$ we mean an analytic subvariety of an open subset of $\mathbf{P}^{s}$. With the Fubini-Study metric, $\mathbf{P}^{s}$ is a Kaehler manifold and there is a natural notion [4] of the $2 k$-volume of a local $k$-variety $W$ of $\mathbf{P}^{s}$, denoted by $2 k$-vol $W$. We have the

Generalized Crofton's formula. Let $W$ be a local $k$-variety in $\mathbf{P}^{s}$. Then

$$
2 k-\operatorname{vol} W=C \int_{\tilde{G}(s, s-k)} n_{W}(\tilde{J}) d \tilde{J}
$$

where $n_{W}(J)=$ the number of points in $W \cap J$ and $C$ is a constant of normalization.

For the proof see de Rham [4] and Santaló [12] (also cf. Chern [3]).

Define a (1,1)-form $\alpha=i / 2 \partial \partial \bar{l} \log \|z\|^{2}$ in $C^{n} \backslash\{0\}$ and put $\alpha_{p}=(1 / p !) \alpha \wedge \ldots$ $\wedge \alpha$ ( $p$ times). We shall apply the following result of Kneser [9] and Lelong [10].

Lemma 2.2. Under the hypotheses of Theorem 2.1, $2 k$-vol $V=\int_{V} \alpha_{k}$.

Proof of Theorem 2.1. If $\omega$ is the $(1,1)$-form on $P^{n-1}$ induced by the Kaehler metric, then $\omega^{p}$ is the volume element (up to normalization) for local $p$ varieties in $\mathrm{P}^{n-1}[4]$. Also $\alpha=\rho^{*}(\omega)\left(\rho: \mathbf{C}^{n} \backslash\{0\} \rightarrow \mathrm{P}^{n-1}\right)$ and so $\alpha^{p}=\rho^{*}\left(\omega^{p}\right)$.

Now $\rho \mid V$ has discrete fibers (as $0 \notin V$ ) and therefore rank $\rho \mid V=k$ on each component of $V$. Thus there is a subvariety $A$ of $V$ of dimension $<k$ such that $V \backslash A$ consists of regular points of $V$ at which rank $\rho \equiv k$. Write $V \backslash A$ $=U\left\{V_{m}: 1 \leq m<\infty\right\}$ where $V_{m}$ is a coordinate neighborhood in $V$ on which $\rho$ maps biholomorphically onto a local $k$-variety in $\mathbf{P}^{n-1}$. Put $S_{m}=V_{m} \backslash \cup\left\{V_{j}: 1 \leq j\right.$ $<m$ \}. Letting $C_{1}, C_{2}, C_{3}$ be constants of normalization we get

$$
\begin{aligned}
2 k \text {-vol } V & =C_{1} \int_{V} \alpha^{k} \quad(\text { Lemma 2.2) } \\
& =C_{1} \int_{V \backslash A} \alpha^{k}=C_{1} \sum_{m=1}^{\infty} \int_{S_{m}} \rho^{*}\left(\omega^{k}\right) \\
& =C_{1} \sum_{m=1}^{\infty} \int_{\rho\left(S_{m}\right)} \omega^{k} \quad\left(\left.\rho\right|_{S_{m}} \text { is } 1-1\right) \\
& =C_{2} \sum_{m=1}^{\infty} 2 k \text {-vol }\left(\rho\left(S_{m}\right)\right) \\
& =C_{3} \sum_{m=1}^{\infty} \int_{G(n-1, n-1-k)} n_{\rho\left(S_{m}\right)}(\tilde{J}) d \tilde{J} \quad \text { (Crofton) } \\
& =C_{3} \int_{G(n, n-k)} \sum_{m=1}^{\infty} n_{S_{m}}(J) d J \\
& =C_{3} \int_{G(n, n-k)} n_{V \backslash A}(J) d J .
\end{aligned}
$$


It is easy to see from Crofton's formula that if $W \subseteq \mathbf{P}^{n-1}$ is a countable union of local varieties of dimension $<k$, then $\int n_{W}(\tilde{J}) d \tilde{J}=0$. Since $\rho(A) \subseteq \mathbf{P}^{n-1}$ is contained in such a set $W, \int n_{A}(J) d J=\int n_{\rho(A)}(J) d J=0$. Thus $n_{V \backslash A}=n_{V}$ almost everywhere on $G(n, n-k)$ and we conclude that

$$
2 k \text {-vol } V=C_{3} \int_{G(n, n-k)} n_{V}(J) d J \text {. }
$$

To evaluate $C_{3}$, choose $V$ to be the intersection with $B_{n}$ of a $k$-plane close to the origin. Then $2 k$-vol $V$ is close to $\alpha(k)$, the volume of the unit ball in $C^{k}$. Also, as $n_{V}(J)$ is equal to 1 for most $J$ (as $V$ is near 0 ), the right-hand side of $(2.1)$ is close to $\mathrm{C}_{3} \cdot 1$. We conclude that $C_{3}=\alpha(k)=\pi^{k} \backslash k !$.

3. In this section we obtain the results on uniform algebras which will be our main analytic tool. For the algebra of functions analytic on the unit disc the following theorem was obtained in [2].

Theorem 3.1. Let $A$ be a uniform algebra with maximal ideal space $M$. Let $x_{0}$ be a point of $M$ which is represented by a positive measure $\sigma$ on $M$. Let $f \in A$ be such that $f\left(x_{0}\right)=0$. Then for $t \geq 0$

$$
2 \pi t \cdot \sigma\{x:|f(x)| \geq t\} \leq l\left(\Gamma_{t} \cap f(\delta M)\right)
$$

where $l(\cdot)$ is linear measure on the circle $\Gamma_{t}=\{z \in \mathrm{C}:|z|=t\}$.

Proof. Let $\varepsilon>0$ and put $\gamma=\Gamma_{t} \cap f(\varnothing M)$. Since $l$ is a regular measure on $\Gamma_{t}$, there is a continuous real valued function $h$ of $\Gamma_{t}$ such that $0 \leq h \leq 1, h$ is identically one on a neighborhood of $\gamma$ and $\int_{0}^{2 \pi} h\left(t e^{i \theta}\right) d \theta<t^{-1}(l(\gamma)+\varepsilon)$. Let $u$ be the harmonic extension of $h$ to the interior of $\Gamma_{t}$ and let $v$ be the harmonic conjugate of $u$ with $v(0)=0$. Put $F(z)=u(z)+i v(z)$ for $|z|<t$. Define $F(z)$ for $|z|>t$ by $F(z)=2-\overline{F\left(z^{*}\right)}$ where $z^{*}=t^{2} / \bar{z}$ is the symmetric point [1] of $z$ with respect to $\Gamma_{t}$. Thus $F$ is analytic off $\Gamma_{t}$ and furthermore, since $2-z$ is the symmetric point of $z$ with respect to the line $\operatorname{Re} z=1$, it follows by the reflection principle [1] that $F$ is analytic on $\gamma$ (since $u \mid \Gamma_{t} \equiv 1$ on a neighborhood of $\gamma$ ). Thus $F$ is analytic on a neighborhood of $f(\Delta M)$.

Since $f(\Delta M)$ is the spectrum of $f$ as an element of $A$, it follows from the Gelfand theory that $F \circ f \in A$ and so

$$
F(0)=F \circ f\left(x_{0}\right)=\int F \circ f d \sigma
$$

But

$$
F(0)=u(0)=\frac{1}{2 \pi} \int h\left(t e^{i \theta}\right) d \theta<(2 \pi t)^{-1}(l(\gamma)+\varepsilon)
$$

Taking real parts in (3.2) gives

$$
\int \operatorname{Re}(F \circ f) d \sigma<(2 \pi t)^{-1}(l(\gamma)+\varepsilon) .
$$


Since $0<u(z) \leq 1$ on $|z|<t$, it follows that $1 \leq 2-u(z)$. We conclude that $\operatorname{Re} F>0$ on $f(\triangle M)$ and $\operatorname{Re} F \geq 1$ on $\{z \in f(\Delta M):|z| \geq t\}$. Hence

$$
\begin{aligned}
\int \operatorname{Re}(F \circ f) d \sigma & \geq \int_{\{x:|f(x)| \geq t\}} \operatorname{Re}(F \circ f) d \sigma \geq \int_{\{x:|f(x)| \geq t\}} 1 \cdot d \sigma \\
& =\sigma\{x:|f(x)| \geq t\} .
\end{aligned}
$$

We have $\sigma\{x:|f(x)| \geq t\} \leq(2 \pi t)^{-1}(l(\gamma)+\varepsilon)$. Letting $\varepsilon \rightarrow 0$ gives (3.1).

Remark. The theorem is true in somewhat greater generality. Let $A, \propto M, x_{0}, \sigma$ be as before. Let $f_{1}$ and $f_{2}$ be elements of $A$ with no common zeros in $M$ and such that $f_{1}\left(x_{0}\right)=0$. Define $f: M \rightarrow \bar{C}=\mathbf{C} \cup\{\infty\}$ by

$$
\begin{aligned}
f(x) & =f_{1}(x) / f_{2}(x) & & \text { if } f_{2}(x) \neq 0, \\
& =\infty & & \text { if } f_{2}(x)=0 .
\end{aligned}
$$

In particular $f\left(x_{0}\right)=0$. Then (3.1) holds for this $f$. To see this, define $\gamma$ and $F$ as before. Notice that $F$ is analytic at $\infty$ and thus assumes only finite complex values on $f(\varnothing M)$. The function $F \circ f$ is continuous on $\mathcal{} M$ and once we know that $F \circ f \in A$ we can complete the proof as before. That $F \circ f \in A$ is a consequence of the following general fact about Banach algebras, provided we identify the Riemann sphere $\overline{\mathbf{C}}$ with $\mathbf{P}^{1}$ via $\mathbf{C} \ni z \leftrightarrow[z, 1]$ (and $\infty \leftrightarrow[1,0]$ ) in which case $f$ becomes $f(x)=\left[f_{1}(x), f_{2}(x)\right]$ in homogeneous coordinates.

Lemma. Let $A$ be a commutative Banach algebra with identity. For $a \in A$ let $a$ be the Gelfand transform of a defined on the maximal ideal space of $A$. Let $a_{1}$, $a_{2} \cdots, a_{n} \in A$ be such that $\hat{a}_{1}, \hat{a}_{2}, \ldots, \hat{a}_{n}$ have no common zeros on $M$. Let $x \mapsto\left[\hat{a}_{1}(x), \hat{a}_{2}(x), \ldots, \hat{a}_{n}(x)\right]$ define a continuous map $\phi$ of $M$ into $\mathbf{P}^{n-1}$. Let $F$ be analytic on a neighborhood of $\phi(\mathcal{M})$ in $\mathrm{P}^{n-1}$. Then there exists $b \in A$ such that $\hat{b}=F \circ \phi$.

Proof. By assumption, the joint spectrum $\sigma\left(a_{1}, a_{2}, \ldots, a_{n}\right)$ of $a_{1}, a_{2}, \ldots, a_{n}$ does not contain the origin of $\mathbf{C}^{n}$. Hence $\left(z_{1}, z_{2}, \ldots, z_{n}\right) \mapsto\left[z_{1}, z_{2}, \ldots, z_{n}\right]$ defines an analytic map $\rho$ of a neighborhood of $\sigma\left(a_{1}, a_{2}, \ldots, a_{n}\right)$ into $\mathrm{P}^{n-1}$ with $\rho\left(\sigma\left(a_{1}, a_{2}, \ldots, a_{n}\right)\right)=\phi(\varnothing M)$. Thus $F \circ \rho$ is an analytic function on a neighborhood of $\sigma\left(a_{1}, a_{2}, \ldots, a_{n}\right)$. By a theorem of Arens and Calderon [15, p. 63] there is $b \in A$ such that $\hat{b}=F \circ \rho\left(\hat{a}_{1}, \hat{a}_{2}, \ldots, \hat{a}_{n}\right)=F \circ \phi$.

To apply the theorem we utilize the following $[8$, p. 421]

Lemma 3.2. Let $\mu$ be a positive $\sigma$-finite measure on a set $X$. Let $f$ be a positive measurable function on $X$ with distribution function $M(t)=\mu\{x:|f(x)| \geq t\}$, $t \geq 0$. Let $\phi$ be a real valued $C^{1}$ increasing function on $[0, \infty)$ with $\phi(0)=0$. Then

$$
\int_{X} \phi \circ f d \mu=\int_{0}^{\infty} \phi^{\prime}(t) M(t) d t
$$


Corollary 3.3. Let $f$ be as in Theorem 3.1. Then

$$
\pi \int|f|^{2} d \sigma \leq \text { area }(f(c)) \text {. }
$$

Proof. We have $2 \pi t M(t) \leq \int_{f(\mathcal{L}) \cap \Gamma_{i}} t d \theta$ where $M$ is the distribution function of $|f|$ with respect to $\sigma$. Integrating with respect to $t$ gives

$$
\pi \int_{0}^{\infty} 2 t M(t) d t \leq \iint_{f(\mathcal{M})} t d t d \theta
$$

Lemma 3.2 with $\phi(t)=t^{2}$ gives the corollary.

The next corollary deals with areas in $\mathbf{P}^{\prime}$. We identify $\mathbf{C}$ with $\mathbf{P}^{\prime} \backslash\{[1,0]\}$ by $z \leftrightarrow[z, 1]$. The invariant normalized measure on $\mathbf{P}^{\prime}$, which we shall denote by $\nu$, becomes $\left[\pi^{-1} t /\left(1+t^{2}\right)^{2}\right] d t d \theta$ on $C$ where $(t, \theta)$ are polar coordinates.

Corollary 3.4. Let $A, \propto M, x_{0}, \sigma$ be as in Theorem 3.1. Let $f_{1}$ and $f_{2}$ belong to $A$ and have no common zeros on $M$ and suppose $f_{1}\left(x_{0}\right)=0$. Define $f: M \rightarrow \mathbf{P}^{\prime}$ by $f(x)=\left[f_{1}(x), f_{2}(x)\right]$. Then

$$
\int \frac{\left|f_{1}\right|^{2}}{\left|f_{1}\right|^{2}+\left|f_{2}\right|^{2}} d \sigma \leq \nu(f(-M)) .
$$

Proof. By the remark to Theorem 3.1 we have for $t \geq 0$ :

$$
2 \pi t M(t) \leq t \int_{f(\mathcal{L}) \cap \mathrm{I}_{t}} d \theta
$$

where $M(t)$ is the distribution of $|f|$ viewed as a map to $\bar{C}$; i.e., $f(x)$ $=f_{1}(x) / f_{2}(x)$. Multiplying by $\left(\pi\left(1+t^{2}\right)^{2}\right)^{-1}$ and integrating, we get

$$
\int_{0}^{\infty} \frac{2 t}{\left(1+t^{2}\right)^{2}} M(t) d t \leq \iint_{f(\mathcal{M})} \frac{t}{\pi\left(1+t^{2}\right)^{2}} d t d \theta .
$$

Using $\phi(t)=t^{2} /\left(1+t^{2}\right)$ in Lemma 3.2 gives

$$
\int \frac{|f|^{2}}{1+|f|^{2}} d \sigma \leq \nu(f(\Delta M))
$$

As $|f|^{2} /\left(1+|f|^{2}\right)=\left|f_{1}\right|^{2} /\left(\left|f_{1}\right|^{2}+\left|f_{2}\right|^{2}\right)$ we have the corollary.

4. The main step in studying the image sets in Grassmannians is

Theorem 4.1. Let $n \geq 2$ be an integer. There exists a strictly positive decreasing continuous function $c(r)$ defined for $0<r<1$ with the following property: Let $X$ be a compact polynomially convex subset of the closed unit ball in $\mathbf{C}^{n}$ for which the Shilov boundary of $P(X)$ lies in the unit sphere $\left\{z \in \mathbf{C}^{n}:\|z\|=1\right\}$. Let $0<r<1$ be the distance from $X$ to the origin. Let $S=\{J \in G(n, n-1): J \cap X \neq \varnothing\}$. Then the measure of $S$ in $G(n, n-1)$ is bounded below by $c(r)$. 
Corollary 4.2. Let $V$ be a subvariety of $B_{n}$ without isolated points and which does not contain the origin. Then the conclusion of Theorem 4.1 holds for $V$ in place of $X$.

Proof. Let $X_{s}=s^{-1}\left(V \cap s \bar{B}_{n}\right)$ for $r<s<1$ (where $\lambda K=\{\lambda x: x \in K\}$ for $\left.K \subseteq \mathbf{C}^{n}, \lambda \in \mathbf{C}\right)$. Then the set of $J \in G(n, n-1)$ which meet $V$ contains the set of $J$ which meet $X_{s}$ and the latter set has measure $\geq c(r / s)$ by the theorem, since $r / s$ is the distance from $X_{s}$ to the origin. Letting $s \rightarrow 1$ gives the desired conclusion.

We begin the proof of Theorem 4.1 with some remarks on $G(n, n-1)$. Let $e_{n}$ be a fixed unit vector in $\mathrm{C}^{n}$ such that $r e_{n} \in X$. Then $\left[e_{n}\right]^{\perp}$, the orthogonal complement of the C-linear span $\left[e_{n}\right]$ of $e_{n}$, is an element of $G(n, n-1)$. Put $G^{\prime}=G(n, n-1) \backslash\left\{\left[e_{n}\right]^{\perp}\right\}$. We shall identify $P^{n-2}$ with the set of complex lines in $\left[e_{n}\right]^{\perp}$ and we define a map $\phi: G^{\prime} \rightarrow P^{n-2}$ as follows: If $J \in G^{\prime}$, let $\pi_{J}$ be the orthogonal projection map of $\mathbf{C}^{n}$ onto $J$. Then $J=\pi_{J}\left(\left[e_{n}\right]\right) \oplus J^{\prime}$ where $\pi_{J}\left(\left[e_{n}\right]\right)$ $\perp J^{\prime}$ and $J^{\prime}$ is an $n-2$ dimensional subspace of $\left[e_{n}\right]^{\perp}$. We set $\phi(J)$ equal to the orthogonal complement of $J^{\prime}$ in $\left[e_{n}\right]^{\perp}$. Thus $\phi(J)$ is a complex line in $\left[e_{n}\right]^{\perp}$; i.e., $\phi(J) \in \mathbf{P}^{n-2}$. The fibers $\phi^{-1}(u), u \in \mathbf{P}^{n-2}$, can be naturally identified to $\mathbf{P}^{\mathbf{l}}$ with a point deleted: In fact, if $u \in \mathbf{P}^{n-2}$, then $u \oplus\left[e_{n}\right]$ is a two-dimensional subspace of $\mathbf{C}^{n}$ and $\phi^{-1}(u)=\left\{J \in G^{\prime}: J=b \oplus\left(u \oplus\left[e_{n}\right]\right)^{\perp}\right.$ where $b$ is any complex line in $u \oplus\left[e_{n}\right]$ other than $\left.u\right\}$. Thus, associating $J$ with $b$ gives an identification of $\phi^{-1}(u)$ with $\mathbf{P}^{\prime} \backslash\{\infty\}$. Let $\beta_{u}$ be the differential 2 -form on $\phi^{-1}(u)$ obtained by restricting the invariant volume form (of total mass one) of $\mathbf{P}^{\mathbf{l}}$ to $\mathbf{P}^{\prime} \backslash\{\infty\}$ $=\phi^{-1}(u)$ via the above identification. Let $\omega$ be the invariant volume form on $\mathbf{P}^{n-2}$. Finally we define a differential form $\alpha$ on $G^{\prime}$ by $J \rightarrow \beta_{\phi(J)} \wedge \phi^{*}(\omega)$. If $\gamma$ is the invariant volume form on $G(n, n-1)$, then $\alpha$ is pointwise on $G^{\prime}$ a positive multiple of $\gamma$. Hence if $m_{1}$ is the regular positive Borel measure induced (on $G^{\prime}$ and hence) on $G(n, n-1)$ by $\alpha$ and $m_{2}$ by $\gamma, m_{1}$ is absolutely continuous with respect to $m_{2}\left(m_{1}<<m_{2}\right)$. Also $m_{1}$ is a finite measure; for by the Fubini-Tonelli theorem

$$
\begin{aligned}
m_{1}(G(n, n-1)) & =\int_{G^{\prime}} \beta_{\phi(J)} \wedge \phi^{*}(\omega) \\
& =\int_{\mathbf{P}^{n-2}}\left(\int_{\phi^{-1}(u)} \beta_{u}\right) \omega=\int_{\mathbf{P}^{n-2}} 1 \cdot \omega=1
\end{aligned}
$$

Thus we can apply the following fact from measure theory [6, p. 125].

Lemma 4.3. Let $m_{1}<<m_{2}$ be finite positive measures. Then there exists an increasing continuous function $\eta$ defined for $t \geq 0$ such that $\eta(0)=0, \eta(t)>0$ if $t>0$ and $m_{2}(E) \geq \eta\left(m_{1}(E)\right)$ for every measurable set $E$.

Now if $S$ is defined as in Theorem 4.1, our object is to find $c(r)$ such that $m_{2}(S) \geq c(r)$. We shall show below that there is a strictly positive decreasing continuous function $c_{1}(r)$ such that $m_{1}(S) \geq c_{1}(r)$. By Lemma 4.3, we may choose $c=\eta \circ c_{1}$ to complete the proof of Theorem 4.1. 
It remains now to find $c_{1}(r)$. Put $S(u)=S \cap \phi^{-1}(u)$ for $u \in \mathbf{P}^{n-2}$. By FubiniTonelli,

$$
m_{1}(S)=\int_{\mathrm{P}^{n-2}}\left(\int_{S(u)} \beta_{u}\right) \omega
$$

Put $\psi(u)=\int_{S(u)} \beta_{u}$. Identifying $\phi^{-1}(u)$ with the set of complex lines in $u \oplus\left[e_{n}\right]$, we see that $b \in S(u)$ if and only if $b \oplus\left(u \oplus\left[e_{n}\right]\right)^{\perp}$ meets $X$; if and only if $X$ contains a point whose orthogonal projection into $u \oplus\left[e_{n}\right]$ lies on $b$. Thus $S(u)$ is identified with the image of $X$ in the projective space of complex lines of $u \oplus\left[e_{n}\right]$. We have the following estimate for $\psi(u)$ (= the measure of $S(u)$ ).

Lemma 4.4. There is a strictly positive decreasing continuous function $\delta$ defined for $0<r<1$ with the following property: Let $\left\{e_{k}\right\}_{1}^{n-1}$ be an orthonormal basis of $\left[e_{n}\right]^{\perp}$. Then

$$
\sum_{k=1}^{n-1} \psi\left(\left[e_{k}\right]\right) \geq \delta(r)
$$

Assuming this for the moment, we finish the proof of Theorem 4.1. Note that (4.1) holds for every orthonormal basis for $\left[e_{n}\right]^{\perp}$. Hence if $e_{1}^{0}, e_{2}^{0}, \ldots, e_{n-1}^{0}$ is a fixed orthonormal basis of $\left[e_{n}\right]^{\perp}$ and $U$ is any element of the group $u$ of unitary transformations of $\left[e_{n}\right]^{\perp}$, we get $\sum_{k=1}^{n-1} \psi\left(U\left[e_{k}^{0}\right]\right) \geq \delta(r)$. Now integrating over $u$ gives

$$
\sum_{k=1}^{n-1} \int_{u} \psi\left(U\left[e_{k}^{0}\right]\right) d U \geq \delta(r)
$$

But if $u$ is any element of $\mathbf{P}^{n-2}$, the invariance of the measure on the unitary group $u$ implies that for $1 \leq k \leq n-1$

$$
\int_{u} \psi\left(U\left[e_{k}^{0}\right]\right) d U=\int_{u} \psi(U u) d U
$$

Hence we get, for all $u \in \mathbf{P}^{n-2}$,

$$
(n-1) \int_{\mathcal{u}} \psi(U u) d U \geq \delta(r)
$$

Integrating over $\mathbf{P}^{n-2}$ and interchanging the order of integration yields

$$
\int_{u}\left(\int_{p^{n-2}} \psi(U u) d u\right) d U \geq \frac{\delta(r)}{n-1}
$$

By the invariance of the measure in $\mathbf{P}^{n-2}$ with respect to unitary transformations, the inner integral above equals $\int \psi(u) d u$ and thus we get

$$
\int \psi(u) d u \geq \frac{\delta(r)}{n-1}
$$


Putting $c_{1}=\delta /(n-1)$ and observing that

$$
\int_{\mathrm{P}^{n-2}} \psi(u) d u=\int_{\mathrm{P}^{n-2}}\left(\int_{S(u)} \beta_{u}\right) \omega
$$

gives

$$
\int_{P^{n-2}}\left(\int_{S(u)} \beta_{u}\right) \omega \geq c_{1}(r)
$$

as desired. Now it remains only to prove Lemma 4.4.

Let $\left\{e_{k}\right\}_{1}^{n-1}$ be a fixed orthonormal basis for $\left[e_{n}\right]^{\perp}$. Let $z_{k}$ be the $k$ th coordinate function with respect to $\left\{e_{k}\right\}$; i.e., $z=\sum_{1}^{n} z_{k} e_{k}$ for $z \in \mathbf{C}^{n}$. We have seen that $S(u)$ is identified with the set of complex lines in $u \oplus\left[e_{n}\right]$ which meet the orthogonal projection of $X$ into $u \oplus\left[e_{n}\right]$. Thus if $u=e_{k}$ for $1 \leq k \leq n-1$, $S\left(\left[e_{k}\right]\right)$ is identified to the image in $\mathbf{P}^{1}$ of the map $X \backslash\left[e_{k}\right] \ni x \mapsto\left[z_{k}(x), z_{n}(x)\right]$. Fix $r_{0}=1 / \sqrt{ } 2$. We shall show that the function

$$
\delta(r)= \begin{cases}r_{0}\left(1-r_{0}\right) /\left(1+r_{0}\right), & 0<r<r_{0}, \\ r(1-r) /(1+r), & r_{0} \leq r<1,\end{cases}
$$

works in (4.1).

Assume, to begin, that $r \geq r_{0}$. Put $t=(1-r) / r$. Define $Y=\{z \in X$ : $\left.\sum_{1}^{n-1}\left|z_{k}\right|^{2} \leq t\left|z_{n}\right|^{2}\right\}$. By the local maximum modulus principle [5], the Shilov boundary $\Sigma$ of $P(Y)$ is contained in the intersection of $Y$ with the union of the unit sphere and the set $\left\{z: \sum_{1}^{n-1}\left|z_{k}\right|^{2}=t\left|z_{n}\right|^{2}\right\}$. Also $x_{0}=r e_{n} \in Y$ as $x_{0} \in X$. We claim that $z_{n}$ is invertible in $P(Y)$. To see this we argue by contradiction and suppose otherwise. Then $z_{n}$ has a zero on $\hat{Y}$, the polynomially convex hull of $Y$, say at $a=\sum_{1}^{n-1} a_{k} e_{k}$. As $\hat{Y} \subseteq X,\|a\|^{2}=\sum_{1}^{n-1}\left|a_{k}\right|^{2} \geq r^{2}$. Let $\mu$ be a representing measure on $\Sigma$ for the point $a$. We have $a_{k}^{2}=\int z_{k}^{2} d \mu$ for $1 \leq k \leq n-1$. Hence $r^{2} \leq\|a\|^{2} \leq \int \sum_{1}^{n-1}\left|z_{k}\right|^{2} d \mu \leq \int t\left|z_{n}\right|^{2} d \mu \leq t$. This is a contradiction, as $r^{2}>(1-r) / r=t$ by choice of $r_{0}$.

Let $\sigma$ be a representing measure for $x_{0}$ on $\Sigma$ for the algebra $P(Y)$. Let $Q=\left\{z \in \Sigma: \sum_{1}^{n-1}\left|z_{k}\right|^{2}=t\left|z_{n}\right|^{2}\right\}$. We shall estimate $\sigma(Q):$ As $z_{n}$ is invertible in $P(Y), r^{-2}=\int z_{n}^{-2} d \sigma$. This implies $r^{-2} \leq \int\left|z_{n}\right|^{-2} d \sigma=\int_{Q}+\int_{\Sigma \backslash Q}$. On $Q$ (in fact, on $Y),\left|z_{n}\right|^{2} \geq r^{2} /(1+t)$, and on $\Sigma \backslash Q,\left|z_{n}\right|^{2} \geq 1 /(1+t)$. We get $r^{-2} \leq$ $r^{-2}(1+t) \sigma(Q)+(1+t)(1-\sigma(Q))$. It follows that

$$
\sigma(Q) \geq\left(1-(1+t) r^{2}\right) /(1+t)\left(1-r^{2}\right) .
$$

Now we apply Corollary 3.4 to $z_{k} / z_{n} \in P(Y)$ and conclude that

$$
\int \frac{\left|z_{k}\right|^{2}}{\left|z_{k}\right|^{2}+\left|z_{n}\right|^{2}} d \sigma \leq \nu\left[\left(\frac{z_{k}}{z_{n}}(\hat{Y})\right)\right] .
$$


As $Y \subseteq X$, the right-hand side of (4.4) is dominated by $\psi\left(\left[e_{k}\right]\right)$. Summing over $k$ in (4.4) yields

$$
\int \sum_{k=1}^{n-1}\left|z_{k}\right|^{2} /\left(\left(\sum_{k=1}^{n-1}\left|z_{k}\right|^{2}\right)+\left|z_{n}\right|^{2}\right) d \sigma \leq \sum_{k=1}^{n-1} \psi\left(\left[e_{k}\right]\right) .
$$

Now the left-hand side decreases if we integrate only over $Q$, a set on which the integrand is identically equal to $t /(1+t)$. We obtain $(t /(1+t)) \sigma(Q)$ $\leq \sum_{1}^{n-1} \psi\left(\left[e_{k}\right]\right)$. Using (4.3) and simplifying we have

$$
\frac{r(1-r)}{(1+r)} \leq \sum_{k=1}^{n-1} \psi\left(\left[e_{k}\right]\right)
$$

This proves the lemma for $r_{0} \leq r<1$. If $0<r<r_{0}$, put $\lambda=r / r_{0}$ and $X^{\prime}$ $=\lambda^{-1}\left(X \cap \lambda \bar{B}_{n}\right)$. Then we have a function $\psi$ defined with respect to $X$ and $\psi^{\prime}$ with respect to $X^{\prime}$ and clearly $\psi^{\prime} \leq \psi$. Since the distance from $X^{\prime}$ to the origin is $r_{0}$ the previous work implies $\sum_{1}^{n-1} \psi^{\prime}\left(\left[e_{k}\right]\right) \geq r_{0}\left(1-r_{0}\right) /\left(1+r_{0}\right)$. Hence $\sum_{1}^{n-1} \psi\left(\left[e_{k}\right]\right) \geq r_{0}\left(1-r_{0}\right) /\left(1+r_{0}\right)$. Thus $\delta$ as defined in (4.2) satisfies Lemma 4.4.

5. We can now prove the result discussed in the introduction.

Theorem 5.1. Let $1 \leq k<n$ be given. There exists a strictly positive decreasing continuous function $q$ defined for $0<r<1$ with the following property: Let $V$ be $a$ pure $k$ dimensional complex analytic subvariety of $B_{n}$ which does not contain the origin. Let $0<r<1$ be the distance from $V$ to the origin. Then the measure in $G(n, n-k)$ of $S=\{J \in G(n, n-k): J \cap V \neq \varnothing\}$ is bounded below by $q(r)$.

For the case $k=1$, this is simply Corollary 4.2 . We shall also reduce the general case to this corollary. Thus we assume $k>1$ and set $j=n-k, 1 \leq j$ $\leq n-2$.

We begin with some remarks on $G(n, j)$. Let $\left\{e_{k}\right\}_{1}^{n}$ be an orthonormal basis of $\mathrm{C}^{n}$ such that $r e_{n} \in V$. Let $A=\left\{J \in G(n, j): e_{n} \in J\right\}$. Then $A$ is a proper subvariety of $G(n, j)$ and consequently is of measure zero in $G(n, j)$. Let $G^{\prime}=G(n, j) \backslash A$ and identify $G(n-1, j)$ with the set of all $j$ dimensional C-linear subspaces of $\left[e_{n}\right]^{\perp}$. We define a map $\phi: G^{\prime} \rightarrow G(n-1, j)$ as follows: $\phi(J)$ equals the orthogonal projection of $J$ to $\left[e_{n}\right]^{\perp}$; since $e_{n} \notin J$, this projection has dimension $j$. If $J^{\prime} \in G(n-1, j)$, the fiber $\phi^{-1}\left(J^{\prime}\right)$ consists of those $J \in G^{\prime}$ whose orthogonal projections on $\left[e_{n}\right]^{\perp}$ equal $J^{\prime}$; i.e., $\phi^{-1}\left(J^{\prime}\right)$ consists of all $j$ dimensional subspaces of $J^{\prime} \oplus\left[e_{n}\right]$ which do not contain $e_{n}$. If we identify the set of all $j$ dimensional subspaces of $J^{\prime} \oplus\left[e_{n}\right]$ with $G(j+1, j)$ we see that $\phi^{-1}\left(J^{\prime}\right)$ is identified to $G(j+1, j) \backslash A\left(J^{\prime}\right)$ where $A\left(J^{\prime}\right)$ is a proper subvariety of $G(j+1, j)$. Let $\beta_{J}$, be the differential form on $\phi^{-1}\left(J^{\prime}\right)$ obtained by restricting the invariant volume form on $G(j+1, j)$ to $\phi^{-1}\left(J^{\prime}\right)$ via the above identification. Let $\omega$ be the invariant volume form on $G(n-1, j)$ and let $\alpha$ be the differential form on $G^{\prime}$ defined by $J \rightarrow \beta_{\phi(J)} \wedge \phi^{*}(\omega)$. Let $\gamma$ be the invariant volume form on $G(n, j)$. Then $\alpha$ is pointwise on $G^{\prime}$ a positive multiple of $\gamma$. Consequently, if $m_{1}$ is the 
positive regular Borel measure induced (on $G^{\prime}$ and hence) on $G(n, j)$ by $\alpha$ and $m_{2}$ by $\gamma$ then $m_{1}<<m_{2}$. Arguing with the Fubini-Tonelli theorem as in $\S 4$, we see that $m_{1}$ is a finite measure and so Lemma 4.3 applies. Thus in order to prove the assertion of Theorem 5.1 it is enough to show that $m_{1}(S) \geq q_{1}(r)$ where $q_{1}$ is a strictly positive decreasing continuous function. By Fubini-Tonelli,

$$
\begin{aligned}
m_{1}(S) & =\int_{S} \beta_{\phi(J)} \wedge \phi^{*}(\omega) \\
& =\int_{G(n, j-1)}\left(\int_{S \cap \phi^{-1}\left(J^{\prime}\right)} \beta_{J^{\prime}}\right) \omega .
\end{aligned}
$$

We shall show that, for all $J^{\prime} \in G(n-1, j)$,

$$
\int_{S \cap \phi^{-1}\left(J^{\prime}\right)} \beta_{J^{\prime}} \geq c(r)
$$

where $c$ is given by Corollary 4.2. It follows by integrating $(5.1)$ over $G(n-1, j)$ that we may choose $q_{1}(r)$ to be $c(r)$.

In order to verify (5.1) for a fixed $J^{\prime}$, put $V\left(J^{\prime}\right)=V \cap\left(J^{\prime} \oplus\left[e_{n}\right]\right)$ where $V$ is the given subvariety of $B_{n}$ and identify $J^{\prime} \oplus\left[e_{n}\right]$ with $C^{j+1}$. Then $V\left(J^{\prime}\right)$ is identified with a subvariety of $B_{j+1}$ (without isolated points since in $B_{n}$, $\operatorname{dim} V=k$ and $\left.\operatorname{dim}\left(J^{\prime} \oplus\left[e_{n}\right]\right)=j+1=n-k+1\right)$. Also the distance from $V\left(J^{\prime}\right)$ to the origin is $r$ since $r e_{n} \in V\left(J^{\prime}\right)$. By Corollary 4.2, applied to $V\left(J^{\prime}\right) \subseteq B_{j+1}$, the measure of the set of $j$ dimensional subspaces of $J^{\prime} \oplus\left[e_{n}\right]$ which meet $V\left(J^{\prime}\right)$ is at least $c(r)$. But by definition of $\beta_{J}$, this measure is precisely the integral on the left-hand side of (5.1).

6. We shall now obtain some applications of the previous results to normal families. Recall that a family $\exists$ of analytic functions on a complex manifold $\Omega$ is normal if every sequence in $\Im$ has a subsequence which converges uniformly on compact subsets of $\Omega$ either to an analytic function or to $\infty$, and that $\Im$ is normal at a point $x \in \Omega$ if there exists a neighborhood $W$ of $x$ such that the restriction of $\exists$ to $W$ is normal. For $A \subseteq B_{n}$ we shall denote the distance from $A$ to the origin by $d(A, 0)$ with the convention that $d(\varnothing, 0)=1$.

Proposition 6.1. Let $\left\{V_{j}\right\}$ be a sequence of subvarieties of pure dimension $k(0<k<n)$ in $B_{n}$ none of which contain the origin.

(a) If $\lim \inf d\left(V_{j}, 0\right)<R<1$ then there exists an $n-k$ dimensional $\mathbf{C}$-linear subspace $L$ of $\mathbf{C}^{n}$ such that $\lim \inf d\left(V_{j} \cap L, 0\right)<R$.

(b) If $\lim \inf d\left(V_{j}, 0\right)=0$ then there exists $L$ as in (a) such that

$$
\lim \inf d\left(V_{j} \cap L, 0\right)=0 .
$$

Proof. (a) Let $W_{j}=V_{j} \cap\left(R B_{n}\right)$ and $W_{j}^{\prime}=R^{-1} W_{j}$, a subvariety of $B_{n}$ with $d\left(W_{j}^{\prime}, 0\right)=R^{-1} d\left(V_{j}, 0\right)$ (at least if $W_{j}$ is nonempty). Hence $\lim \inf d\left(W_{j}^{\prime}, 0\right)$ $=R^{-1} \lim \inf d\left(V_{j}, 0\right)=\delta$ say, where $0<\delta<1$. If $S_{j}$ is the set of elements of $G(n, n-k)$ which meet $W_{j}^{\prime}$, it follows from Theorem 5.1 that $\lim \sup v\left(S_{j}\right)$ $\geq q(\delta)$ where $\nu$ is invariant measure on $G(n, n-k)$. Since $\nu$ is a finite measure, 
we conclude that there is a point $L$ in infinitely many of the $S_{j}$. Thus $L$ meets infinitely many of the $W_{j}^{\prime}$ and consequently, $L$ meets infinitely many of the $V_{j}$ within $\{z:\|z\|<R\}$.

(b) We may assume, by passing to a subsequence, that $r_{j}=d\left(V_{j}, 0\right) \rightarrow 0$ and $r_{j}<\frac{1}{2}$. Let $W_{j}=V_{j} \cap\left(\left(2 r_{j}\right) \cdot\left(B_{n}\right)\right)$ and $W_{j}^{\prime}=\left(2 r_{j}\right)^{-1} W_{j}$. As before, $W_{j}^{\prime}$ is a subvariety of $B_{n}$ and $d\left(W_{j}^{\prime}, 0\right)=\frac{1}{2}$. Defining $S_{j}$ as above we get $\nu\left(S_{j}\right) \geq q\left(\frac{1}{2}\right)$ and so there is an $L$ meeting infinitely many $W_{j}^{\prime}$. This means that $L$ meets $V_{j}$ within $2 r_{j} B_{n}$ for infinitely many $j$, i.e., $\lim \inf d\left(V_{j} \cap L, 0\right)=0$.

Theorem 6.2. Let $\exists$ be a family of analytic functions on $B_{n}$.

(a) If the restriction of $\rightarrow$ to each complex line through the origin is normal, then $\exists$ is normal.

(b) If the restriction of $\rightarrow$ to each complex line through the origin is normal at the origin, then $\exists$ is normal at the origin.

Proof. We shall prove (a) and (b) simultaneously. Let $\left\{f_{j}\right\} \subseteq \mathcal{\Im}$. (i) Suppose $\left\{f_{j}(0)\right\}$ is a bounded sequence. It is enough to prove that $\left\{f_{k}\right\}$ is uniformly bounded on $B(r)=\{z:\|z\| \leq r\}$ for every $r<1$ in case (a) and for some $r<1$ in case (b). We argue by contradiction and suppose otherwise. Then there exist a subsequence $\left\{f_{j_{s}}\right\}$ and points $\left\{z_{s}\right\}$ such that $f_{j_{s}}(0) \neq f_{j_{s}}\left(z_{s}\right) \rightarrow \infty$ where $\left\|z_{s}\right\|<r$ for some fixed $r<1$ in case (a) and $z_{s} \rightarrow 0$ in case (b). Let $V_{s}=\left\{z \in B_{n}\right.$ : $\left.f_{j_{s}}(z) \equiv f_{j_{s}}\left(z_{s}\right)\right\}$. If $L$ is any complex line, $\left\{f_{n}\right\}$ is uniformly bounded on compact subsets of $L$ in case (a) (since $\left\{f_{n}\right\}$ restricted to $L$ is normal) and $\left\{f_{n}\right\}$ is uniformly bounded on some neighborhood of 0 in $L$ in case (b). Consequently $\lim \inf d\left(V_{j} \cap L, 0\right)=1$ for (a), $>0$ for (b). By Proposition 6.1 then, $\lim \inf d\left(V_{j}, 0\right)=1$ for (a), $>0$ for (b). But $z_{s} \in V_{s}$ implies $\lim \inf d\left(V_{s}, 0\right) \leq$ $\lim \inf \left\|z_{s}\right\| \leq r$ for (a), $=0$ for (b). This is a contradiction.

(ii) If $\left\{f_{j}(0)\right\}$ is unbounded, pass to a subsequence and assume $0 \neq f_{j}(0) \rightarrow \infty$. Let $V_{j}=\left\{z \in B_{n}: f_{j}(z)=0\right\}$. For all complex lines $L, f_{j} \rightarrow \infty$ uniformly on compact subsets of $L$ in case (a), uniformly on compact subsets of some neighborhood of 0 in $L$ in case (b). Hence $\lim \inf d\left(V_{j} \cap L, 0\right)=1$ for (a), $>0$ for (b). By Proposition 6.1, lim inf $d\left(V_{j}, 0\right)=1$ for (a), = some $\delta>0$ for (b). Thus $f_{j} \neq 0$ on $B(r)$ for $j \geq j(r)$ where $r$ is any number less than 1 in case (a) and some number less than one in case (b). Put $g_{j}=1 / f_{j}$ in $B(r)$ for $j \geq j(r)$. Since $g_{j}(0) \rightarrow 0$, (i) implies that $\left\{g_{j}\right\}$ is normal on interior $B(r)$ in case (a) and on some neighborhood of 0 in case (b). Hence $g_{n_{j}} \rightarrow g$ uniformly on compact subsets of $\operatorname{int}(B(r))$ in case (a) and on compact subsets of some neighborhood of 0 in case (b), where $g$ is analytic. Since the $g_{j}$ are zero free and $g(0)=0$, it follows that $g \equiv 0$ by a theorem of Hurwitz. Hence $f_{n_{j}} \rightarrow \infty$ uniformly on compact subsets of int $(B(r))$ in case (a), uniformly on compact subsets of some neighborhood of 0 in case (b). This completes (b). The usual diagonal process gives (a).

Corollary 6.3 (Hartogs [7]). Let $F=\sum c_{\alpha} z^{\alpha}$ be a formal power series in $n$ complex variables. If the restriction of $F$ to each complex line through the origin is convergent, then $F$ is convergent. 
Proof. Put $\exists=\left\{f_{m}\right\}$ where $f_{m}=\sum\left\{c_{\alpha} z^{\alpha}:|\alpha| \leq m\right\}$. By the theorem $\Im$ is normal at 0 , from which it follows easily that $F$ is convergent.

\section{REFERENCES}

1. L. Ahlfors, Complex analysis, an introduction to the theory of analytic functions of one complex variable, McGraw-Hill, New York, 1953. MR 14, 857.

2. H. Alexander, B. A. Taylor and J. L. Ullman, Areas of projections of analytic sets, Invent. Math. 16 (1972), 335-341.

3. S. S. Chern, Lectures on integral geometry, Notes by H. C. Hsiao, multilithed, 1965.

4. G. de Rham, On currents in an analytic complex manifold, Seminars on Analytic Functions, vol. 1, Princeton, N.J., 1957, pp. 54-64.

5. T. Gamelin, Uniform algebras, Prentice-Hall, Englewood Cliffs, N.J., 1969.

6. P. Halmos, Measure theory, Van Nostrand, Princeton, N.J., 1950. MR 11, 504.

7. F. Hartogs, Zur Theorie der analytischen Funktionen mehrer unabhangiger Veränderlichen, Math. Ann. 62 (1906), 1-88.

8. E. Hewitt and K. Stromberg, Real and abstract analysis. A modern treatment of the theory of functions of a real variable, Springer-Verlag, New York, 1965. MR 32 \#5826.

9. H. Kneser, Zur Theorie der gebrochenen Funktionen mehrer Verdnderlichen, Jber. Deutsch. Math. Verein. 48 (1938), 1-28.

10. P. Lelong, Intégration sur un ensemble analytique complexe, Bull. Soc. Math. France 85 (1957), 239-262. MR 20 \#2465.

11. T. Nishino, Sur une propriété des familles de fonctions analytiques de deux variables complexes, J. Math. Kyoto Univ. 4 (1965), 255-282.

12. L. Santaló, Integral geometry in Hermitian spaces, Amer. J. Math. 74 (1952), 423-434. MR 13, 971.

13. W. Stoll, About the convergence of a power series, Festschrift zur Gedächtnisfeier für Karl Weierstrass, Westdeutscher Verlag, Berlin, 1966, pp. 523-529. MR 33 \$5662.

14. G. Stolzenberg, Volumes, limits, and extensions of analytic varieties, Lecture Notes in Math., vol. 19, Springer-Verlag, Berlin, 1966. MR 34 \#6156.

15. E. L. Stout, The theory of uniform algebras, Bogden and Quigley, Belmont, Calif., 1971.

Department of Mathematics, University of Michigan, Ann Arbor, Michigan 48104 